\title{
Editorial
}

\section{La Importancia de la Revisión por Pares para Avanzar en Ciencia}

\section{The Importance of the Peer Review to Advance in Science}

\author{
Herney Andrés García-Perdomo, MD, MSc, EdD, PhD, FACS ${ }^{10}$ Hugo Enrique López-Ramos, MD, MSc ${ }^{2}$ \\ ${ }^{1}$ Departamento de Cirugía/Urología. Escuela de Medicina. \\ Universidad del Valle. Cali, Colombia \\ 2 Jefe del servicio de Urología. Hospital San Ignacio. Pontificia \\ Universidad Javeriana. Bogotá, Colombia. \\ Urol Colomb 2021;30:87-88.
}

\section{Introducción}

La revisión por pares es una estrategia que data de mucho tiempo atrás, posiblemente desde la Antigua Grecia. ${ }^{1}$ Es una herramienta que ha apoyado a la Medicina y a la ciencia al intentar regular la calidad de lo descrito por nuestros pares al ser abierta al público.

La estrategia de revisión por pares es un proceso de evaluación del trabajo académico, la investigación o las ideas de un autor al escrutinio de otros, quienes son expertos en la misma área $^{1,2}$ de manera tal que su propósito es mejorar la calidad de los manuscritos que son candidatos a ser publicados, verificar y confrontar su pertinencia y precisión, además de asegurar que solo se publique un trabajo académico de alta calidad.

\section{Ventajas y desventajas}

De manera natural, todas las intervenciones tienen pros y contras. ${ }^{2,3}$ Con respecto a las ventajas, se encuentran: 1) ofrecer comentarios y sugerencias a los autores para mejorar el producto final, así disminuye la posibilidad de presentar errores; 2) ayudar al editor de una revista a decidir si un articulo es aceptable, puesto que los expertos en el área sugieren si un trabajo puede contribuir al avance de la ciencia; 3) ser invitado como revisor es un honor pues sugiere que su trabajo en el área y/o sus habilidades como evaluador de la literatura son respetadas, por tanto, es un trabajo que puede ser usado como credencial de presentación y reconocimiento público; 4) como se dijo previamente, los lectores podrían considerar que se ha realizado un filtro riguroso y la información presentada es la de mejor calidad científica. Sin embargo, no se pueden detectar todos los casos de trabajo fraudulento, investigación pobre o con faltas en la integridad científica; 5) permitir que los evaluadores estén

Address for correspondence Herney Andrés García-Perdomo, MD MSc, EdD, PhD, FACS, Departamento de Cirugía/Urología, Escuela de Medicina, Universidad del Valle, Cali, Colombia

(e-mail: editorrevista@scu.org.co). actualizados con lo último en investigación en el área antes de ser de dominio público. Lo anterior permite estimular nuevas ideas de investigación; y 6) la revisión por pares requiere entrenamiento y estandarización para ser realizada de la mejor manera, por tanto mejora las capacidades de lectura crítica y escritura de los revisores.

Por otro lado, están las desventajas o detractores de esta estrategia: 1) es un proceso riguroso que consume tiempo y no tiene pago; 2) los revisores pueden estar sesgados de acuerdo con sus propios intereses de investigación, conflictos de interés financieros o personales, entre otros. Esto requiere de un análisis mayor en investigación; 3) en diferentes ocasiones se presentan inconsistencia entre dos o más revisiones y recomendaciones, esto lleva a conflictos para el autor y el editor; 4) la revisión por pares puede ser uno de los cuellos de botella más importantes para la publicación. Los revisores no aceptan, o generan apreciaciones superficiales que no apoyan al editor. Por tanto, se retrasa el tiempo a la publicación, llevando a publicaciones con información desactualizada y frustración para el autor.

\section{La revision estructurada}

En general existen múltiples formatos desde los no estructurados, pasando por diferentes estructuras (simples y complejas). Nosotros sugerimos los elementos descritos en PLOS 4 y que describiremos a continuación como parte de nuestra tarea para apoyar a los revisores:

1. Usar una estructura clara, que ayudará tanto al editor como al autor. Se sugiere una pirámide invertida. Esta tendrá la información más importante al inicio, detalles y ejemplos en la mitad, y puntos adicionales finalmente.

2. Con respecto al primer punto, se debe resumir con sus propias palabras lo que el manuscrito quiere ofrecer o

(C) 2021. Sociedad Colombiana de Urología. All rights reserved. This is an open access article published by Thieme under the terms of the Creative Commons Attribution-NonDerivative-NonCommercial-License, permitting copying and reproduction so long as the original work is given appropriate credit. Contents may not be used for commercial purposes, or adapted, remixed, transformed or built upon. (https://creativecommons.org/ licenses/by-nc-nd/4.0/)

Thieme Revinter Publicações Ltda., Rua do Matoso 170, Rio de Janeiro, RJ, CEP 20270-135, Brazil 
reportar. Esto permite conocer la impresión e interpretación que el revisor tuvo del trabajo. Se dará un resumen de las fortalezas y limitaciones del manuscrito. Se debe finalizar esta sección con la sugerencia de acción con respecto al articulo.

3. Debe discutir los puntos de mejora de áreas especificas. Se sugiere la división en dos apartados: elementos mayores y elementos menores. Los elementos mayores son aquellos esenciales para que el manuscrito tenga una apropiada validez interna; los menores son aquellos que no afectaran los resultados y las conclusiones (referencias ausentes, clarificaciones, presentación de datos, corrección de estilo, entre otros). Requiere la justificación de las recomendaciones con evidencia concreta y ejemplos específicos de tal manera que el autor pueda mejorar su manuscrito. No se sugiere recomendar experimentos adicionales que estén fuera del objetivo del trabajo de investigación.

4. Otros elementos generales: el apartado de comentarios confidenciales al editor se usa para informar al editor acerca de elementos de ética, conflictos de interés, o si podría revisar una nueva versión del articulo. No se debe usar este espacio para criticar el manuscrito pues no llegará al autor, ni le ayudará a mejorar el manuscrito.

\section{Recomendaciones}

Aunque existan detractores de este proceso, en la actualidad es requerido por la mayoría de las revistas científicas para permitir una publicación optima y de la mejor calidad posible. Por tanto, ofreceremos algunos elementos para estimular y mejorar el proceso de los revisores en esta ardua pero gratificante tarea para la ciencia. ${ }^{1}$

De acuerdo con Brian Lucey, ${ }^{5}$ existen diez tips para ser un revisor efectivo:

1. Sea profesional. Recuerde que es la responsabilidad con el desarrollo de la ciencia.

2. Sea agradable y respetuoso. Una revisión debe ofrecer respeto por nuestros compañeros clínicos y científicos. No se beneficia de ser rudo (maleducado) en sus comentarios.

3. Lea la invitación. Cuando se invita a una persona a revisar un manuscrito, este viene con unas instrucciones para hacerlo. Lea detenidamente y sígalas.

4. Sea servicial. Los autores deben conocer lo que esta bien y lo que merece mejorar. Sugiera a los autores como puede mejorar la calidad de su articulo.

5. Sea científico. Enfoque sus comentarios en lo que va a mejorar el conocimiento científico e incrementará la credibilidad de la investigación y las conclusiones a las que se han llegado. Si hay errores tipográficos o de estilo, sugiera una revisión profesional de estilo, gramática y escritura.

6. Cumpla los tiempos. Es importante tanto para el editor como para el autor. Esto permitirá darle celeridad y gestión al proceso editorial.

7. Sea realista. En algunas oportunidades, los revisores son muy ambiciosos con los cambios sugeridos a los manuscritos. Se debe ser realista con respecto al trabajo presentado y los cambios a realizar por el autor.

8. Sea empático. Es importante que escriba de manera sensible y respetuosa, de tal manera que su revisión sea científica, cortes y servicial.

9. Sea de mente abierta. Todos los comentarios son importantes, tanto los especializados, los metodológicos como los generalistas. Recuerde que si un editor le ha invitado, significa que confía en su valido rol como evaluador.

10. Sea organizado. La revisión debe ser estructurada y lógica. Existen sugerencias de orden de escritura: sígalas. Además, revise antes de enviar.

En conclusión, la revisión por pares es una estrategia que nos permite mejorar la calidad de la información científica que leemos. Adicionalmente, es una puesta al público del estado actual de la investigación, de tal manera que se requieren otros elementos de integridad científica que apoyen la revisión por pares para confiar en los resultados. La participación en estos tan interesantes e importantes procesos permitirá el crecimiento de nuestra cultura en investigación y la aplicación de la mejor evidencia en la practica clínica.

\section{Referencias}

1 Kelly J, Sadeghieh T, Adeli K. Peer review in scientific publications: benefits, critiques \& a survival guide. EJIFCC 2014;25(03): 227-243

2 Koshy K, Fowler AJ, Gundogan B, Agha RA. Peer review in scholarly publishing part A: why do it? Int J Surg Oncol 2018;3(02):56. DOI: http://doi.org/10.1097/IJ9.0000000000000056

3 Cowell JM. Importance of peer review. J Sch Nurs 2014;30(06): 394-395

4 PLOS. Peer Review Template - PLOS [Internet]. Report. [cited 2021 Apr 10] Available from: https://plos.org/resource/peer-reviewtemplate/

5 The guardian. Peer review: how to get it right - 10 tips [Internet]. Report [cited 2021 Apr 10]. Available from: https://www. theguardian.com/higher-education-network/blog/2013/sep/27/ peer-review-10-tips-research-paper 